\title{
Multiple-well invasion percolation
}

\author{
A. D. Araújo, ${ }^{1}$ M. C. Romeu, ${ }^{1}$ A. A. Moreira, ${ }^{1}$ R. F. S. Andrade, ${ }^{2}$ and J. S. Andrade, Jr. ${ }^{1}$ \\ ${ }^{1}$ Departamento de Física, Universidade Federal do Ceará, 60451-970 Fortaleza, Ceará, Brazil \\ ${ }^{2}$ Instituto de Física, Universidade Federal da Bahia, 40210-340 Salvador, Bahia, Brazil
}

(Received 27 November 2006; revised manuscript received 6 July 2007; published 24 April 2008)

\begin{abstract}
When the invasion percolation model is applied as a simplified model for the displacement of a viscous fluid by a less viscous one, the distribution of displaced mass follows two distinct universality classes, depending on the criteria used to stop the displacement. Here we study the distribution of mass for this process, in the case where four extraction wells are placed around a single injection well in the middle of a square lattice. Our analysis considers the limit where the pressure of the extraction well $P_{e}$ is zero; in other words, an extraction well is capped as soon as less viscous fluid reaches that extraction well. Our results show that, as expected, the probability of stopping the production with small amounts of displaced mass is greatly reduced. We also investigate whether or not creating extra extraction wells is an efficient strategy. We show that the probability of increasing the amount of displaced fluid by adding an extra extraction well depends on the total recovered mass obtained before adding this well. The results presented here could be relevant to determine efficient strategies in oil exploration.
\end{abstract}

DOI: 10.1103/PhysRevE.77.041410

PACS number(s): 61.43.Gt, 47.55.-t, 64.60.-i

\section{INTRODUCTION}

The investigation of single and multiphase flow in a porous media $[1,2]$ profits from several concepts and models stemming from percolation theory in a discrete lattice [3-6]. Despite being a great simplification of the realistic flow of fluids through a porous substrate, in several circumstances (e.g., extraction of oil and gas from underground reservoirs) the prevailing conditions of the flow justifies many of the necessary assumptions to map the problems onto each other. Fixing our attention to the oil exploration scenario, we recall that the first stage of the exploration process is usually called spontaneous production, when then oil flow is generated by the internal pressure of the reservoir. Here we focus on the second stage, when water or some other wetting and less viscous fluid is injected underground to displace the oil from the porous medium.

In a two-phase flow, the contact forces at the two-fluid interface, caused by the pressure gradient pointing from the invading to the displaced fluid, is characterized by the superficial tension $\sigma$, which is combined with the typical velocity $u$ and the viscosity $\mu$ to form the capillary number

$$
\mathrm{Ca} \equiv \frac{u \mu}{\sigma} .
$$

In the extreme limit of Stokes flow where the Reynolds number is low, $\mathrm{Re} \equiv \frac{u L \rho}{\mu} \ll 1, \mathrm{Ca}$ quantifies the properties of the flow in regard to the balance between layer to layer dissipation force (viscosity) and fluid to wall dissipation force (capillary). In the low Ca regime, capillary forces are the most relevant ones, scaling down the penetration problem to be dependent on the local porous geometry; that is, the displacement of the more viscous fluid happens first in the pores where the capillary pressure is lower. In such conditions, the model of nontrapping invasion percolation (NTIP) in a discrete porous lattice captures most of the essential aspects of the forced displacement of a fluid in a porous environment [7]. For example, one aspect of the exploration process that cannot be described by the NTIP model is the fact that water may completely surround regions filled with oil that can no longer be recovered.

The second phase of oil exploration can be modeled within the NTIP framework, with the first site of the percolation cluster at the injection well. The oil displaced by injected water is recovered at the extraction well, which is held at a very low pressure. The process evolves until the water cluster reaches the extraction well, corresponding to the breakthrough time, when the oil production stops. In a previous paper [8], the NTIP model has been used to evaluate the typical amount of displaced fluid that can be recovered from a reservoir which corresponds to the size of the cluster generated in the invasion.

Here we include the possibility of new extraction wells being added to the exploration field at the second phase of the oil extraction. This investigation raises several interesting questions related to possible paths to a more efficient production when more wells are bored in the production field. The first question refers to the decision of working with more than one extraction well simultaneously instead of boring them one at a time, after the previous well has exhausted its production. One can also compute the probability distribution of recovered oil in subsequent wells in comparison to the former ones. Answering these questions in the context of a very simple model may be of relevance in helping to define production strategies, which must take into account the costs of boring further wells.

The rest of this paper is organized as follows. In Sec. II we bring a brief review of the results of the previous work and present a summary of the model that is used in the simulations. In Sec. III, results obtained from the computational simulations are discussed. In Sec. IV we compare the results with those provided by analytical estimates, taking into account the assumption that the joint probability distribution function (PDF) of producing oil from two wells is given by the product of two one-well types of PDF. Finally, in Sec. V, we close the paper with conclusions and discussions on possible applications of our results. 


\section{MODEL}

The basic assumption of the NTIP model for the extraction process is that the square lattice represents a series of connected pores filled with the displaced fluid (e.g., oil). The use of two-dimensional (2D) simulations can be justified when considering narrow oil fields [9-15]. Also, the trapping effects that are more relevant in two dimensions should play a smaller role in three-dimensional systems. The model considers further the $\mathrm{Ca} \rightarrow 0$ limit, which is approximately valid in the situation where water (or other wetting fluid) slowly invades a porous medium containing oil. In this process, the interface between the two fluids in a pore is subject to a pressure difference expressed by $p_{i}$ that depends on the physical properties of the fluid as well as on the geometrical aspects of the pore and will be different to each specific pore $i$. Under the same driving pressure, the pore with smallest capillary pressure, which is typically the pore with the largest volume, is invaded first. Since the advance of the invading front depends only on the order by which the pores are invaded, as long as all correlations are disregarded, the particular distribution of pressures $p_{i}$ is not relevant in this model. This justifies our choice for a simple uniform distribution.

The NTIP algorithm starts by assigning a uniform random distribution of pressures $p_{i}$ in the range $[0,1]$ for each site of the network, mimicking the heterogeneity of the medium. The penetration starts at the injecting well $W_{0}$, where the boundary of the invading front consists initially of just four neighbors. The site with the smaller pressure is invaded and the front boundary is updated. The front boundary is the set of all noninvaded sites that are neighbors of at least one invaded site. The procedure is repeated over and over until a stopping criterion is reached and, at this point, we measure the mass of the invaded cluster.

The propagation front evolves from the injection well to one, or several, extracting wells. In our study, we place the injection well in the center of a square lattice of size $L$ and four extraction wells at a distance $r$ from the central injection well placed along the natural axes of the square lattice. We study the two following exploring strategies: $(A)$ to bore all wells at the same time, and $(B)$ to bore each extracting well only after the oil obtained with the preceding well has been exhausted. Since we have restricted ourselves to the limit where the capillary pressure at the extraction sites is zero, as soon as each extraction site is reached by the growing cluster, the production on this site stops. After that, the exhausted extraction well is capped. Although the final amount of extracted oil is the same in both strategies, strategy $B$ gives the possibility of not boring the following wells depending on the oil recovered in the previous steps. In our study we measure the masses $M_{i}$ produced before each of the four extraction wells is reached. This can be roughly expressed by the number of invaded sites. Thus, in strategy $A$, we define $M_{1}$ as the number of invaded sites until one of the four wells is reached. The number of sites invaded after reaching the first well and before reaching a second well is $M_{2}$ and so on. On strategy $B$, only one of the four wells is opened at the beginning of the process. For this strategy, the total mass of the invaded cluster when this extraction well is reached is defined as $M_{1}$. After the first well is reached, the preassigned

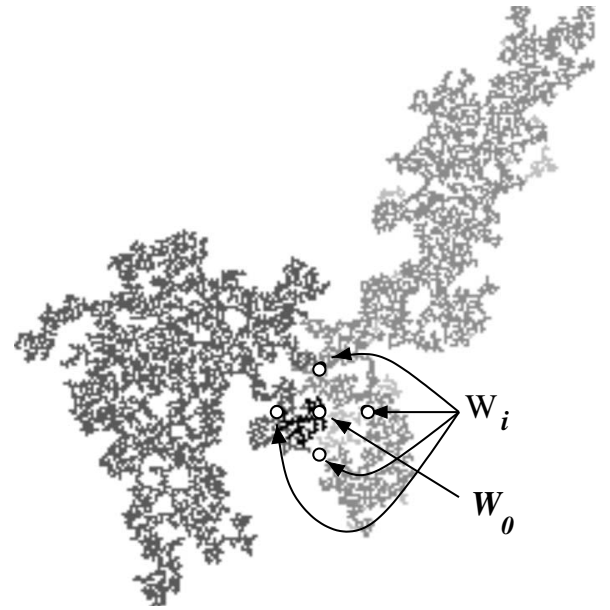

FIG. 1. Time evolution of the invasion process between the injection well $\left(W_{0}\right)$ and the multiple extraction wells $\left(W_{i}\right)$, where $i=1,2,3,4$. The invasion process is identified in accordance with the sequence where the extraction sites are reached. The tones of gray indicate the sites that were incorporated at each iteration of the invasion process, becoming darker from the first to the last well.

site to the second well is opened, and the process continues until it is invaded. However, there is the possibility that, when the second well is opened, it had already been invaded. In this case, the well produces no oil $\left(M_{2}=0\right)$, and the next well can be opened.

This process is repeated for several distinct network realizations in order to obtain the mass distributions of invaded clusters $P_{i}(M), i=1,2,3,4$. It is worth noticing that it is not necessary to produce different realizations for strategies $A$ and $B$, since both can be generated if one keeps track of the order at which the wells have been invaded. Of course, in an actual situation, these procedures are not equivalent due to the difference in cost to open the wells. Therefore, the knowledge of the distinct strategies may be of great relevance.

\section{RESULTS OF SIMULATIONS}

We start by analyzing the results obtained with the exploration strategy $A$. A typical realization of multiple-wells invasion with this strategy is illustrated in Fig. 1. There we show the clusters, in different gray shades, formed when the invading fluid reaches successively wells $1-4$. The entire cluster looks similar to those obtained in a two-well configuration, and has the same fractal dimension of the traditional invasion percolation model [7]. To compute the probability density function of the recovered masses, we perform several realizations of the model and record the mass added to the cluster at each moment of the invasion; that is, before the invasion reaches each of the wells. In order to avoid boundary effects, we discard all events where the cluster touches the boundary of the lattice before reaching the last well. It was shown in Ref. [8] that these events, identified as offcritical clusters, follow a distinct size distribution whose shape depends on the limiting scales of the oil field. For a system size $L=2048$, and well separation $r=32$, we find that 


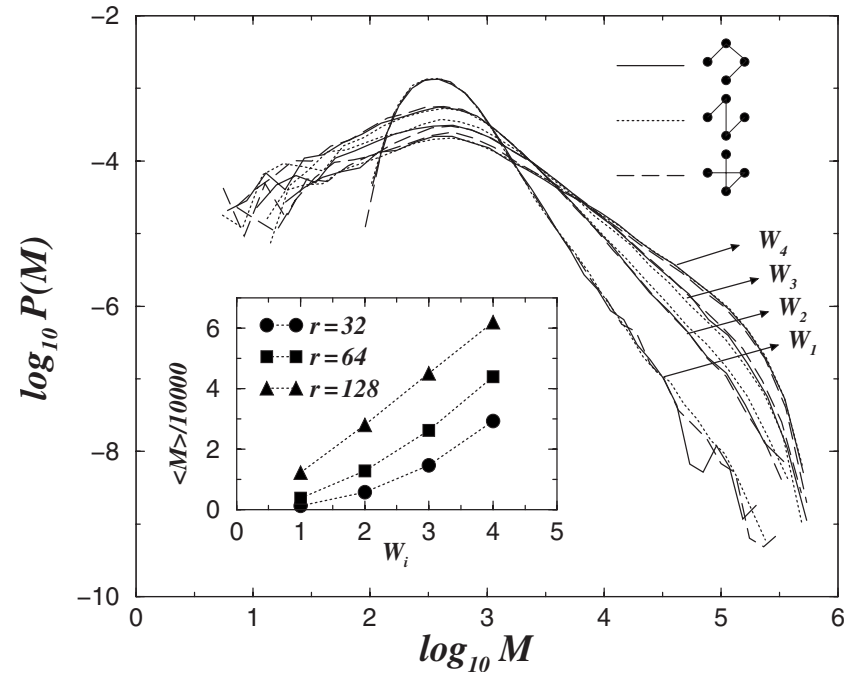

FIG. 2. Graph in log-log scale of the distribution of recovered mass when each of the extraction wells is reached. This figure concerns the exploration strategy $A$ with system size $L=2048$ and well separation $r=32$. The different line stiles in the main panel represent the events where the wells were invaded in one of the possible distinct orders shown in the upper right corner of the figure. It is clear that the distributions do not depend on the particular order by which the wells are reached. The distributions decay as power laws with exponents $\alpha_{1}=2.45 \pm 0.07, \alpha_{2}=1.74 \pm 0.05, \alpha_{3}=1.34 \pm 0.05$, and $\alpha_{4}=1.04 \pm 0.03$ corresponding, respectively, to each well $W_{1}$, $W_{2}, W_{3}$, and $W_{4}$. In the inset we show the average recovered mass as a function of the well rank and for different values of well distance $r$.

less than $3 \%$ of the realizations reach the border without reaching any of the four wells and about $18 \%$ of the realizations reach the four extraction wells without touching the boundary. Despite the fact that realizations reaching the boundary appear for any scale, they become less probable as the ratio $L / r$ increases. This justifies the separate analysis of the distributions for the different sets of clusters. The consequences of off-critical events in the current investigation are much the same as those studied in Ref. [8]. For the sake of avoiding repeating the same discussion, we refrain from inserting herein the details of that distribution, and concentrate only on the distribution of critical clusters.

The shape of the critical cluster size distributions is shown in Fig. 2, where we draw the distribution $P_{i}(M)$ in $\log$-log scale, associated with the cluster mass $M_{i}, i$ $=1,2,3,4$. In this case, the mass $M_{i}$ represents the mass invaded after the well $W_{i-1}$ was reached and capped and before the well $W_{i}$ was reached and capped. The distributions show a consistent power-law behavior $P_{i}(M) \sim M^{-\alpha_{i}}$ in the intermediate range of $M$.

The curves were obtained with $10^{5}$ realizations with a system size $L=2048$, and distance between the invasion well and extraction wells $r=32$. The least-squares fit to the data in the scaling region leads to $\alpha_{1}=2.45 \pm 0.07, \alpha_{2}=1.74 \pm 0.05$, $\alpha_{3}=1.34 \pm 0.05$, and $\alpha_{4}=1.04 \pm 0.03$, corresponding to each well $W_{1}, W_{2}, W_{3}$, and $W_{4}$, respectively. The exponent controlling the decay of the distribution becomes less negative from the first to the last well; that is, the last well to be reached has a higher probability of generating larger values

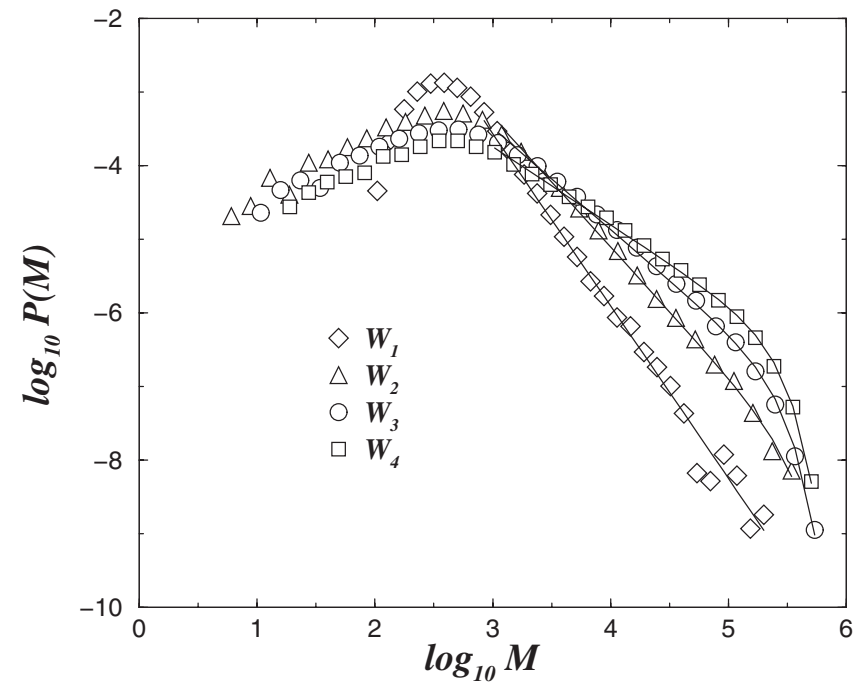

FIG. 3. The distributions of recovered mass for strategy $A$. The system size and well separation are the same as the ones used in Fig. 2. The different symbols represent the order that the wells were invaded. The solid lines correspond to the best fit to the data of the scaling function, Eq. (2). The value obtained for the parameter controlling the onset of the truncation was $A=0.22$. The values of the exponents $\quad \alpha_{i} \quad$ were $\alpha_{1}=2.40 \pm 0.07, \quad \alpha_{2}=1.76 \pm 0.05, \quad \alpha_{3}$ $=1.38 \pm 0.05$, and $\alpha_{4}=1.06 \pm 0.03$ corresponding, respectively, to each well $W_{1}, W_{2}, W_{3}$, and $W_{4}$. These values are within the error bars of those obtained with the simpler fit of that shown in Fig. 2.

of mass. The inset of the same figure shows that the average value of the recovered mass grows with the rank of the extraction well and with the geometrical distance between the wells $r$.

Four wells can be invaded in 24 different sequences, which, by symmetry, can be reduced to the three nonequivalent conformations displayed in Fig. 2. The results presented in Fig. 2 indicate that the shape of the distribution is not affected by the order in which the wells are reached during the extraction process.

Two crossovers can be observed for each distribution function, one at smaller values of mass and another at larger values. As discussed in [8], these crossovers are exponential truncations, whose characteristic values are determined by the geometrical features of the model. In small scales, one sees a cutoff that depends on the well separation distance as $r^{D_{f}}$, where $D_{f}$ is the fractal dimension of the percolation cluster. The effect of changing the distance $r$ between the injection and extraction wells is to shift the range of the scaling region. Similarly, the truncation at large values of mass depends on the lattice size as $L^{D_{f}}$. Based on these features we argue that, for large values of $M_{i}, P\left(M_{i}\right)$ should obey the following scaling ansatz:

$$
P_{i}(M) \sim M^{-\alpha_{i}} \exp \left[-\left(\frac{M}{A L^{D_{f}}}\right)^{2}\right],
$$

where $\alpha_{i}$ are the scaling exponents. In Fig. 3 we fit our distributions with this equation. The values of the exponents we find with the help of Eq. (2) are $\alpha_{1}=2.40 \pm 0.07, \alpha_{2}$ $=1.76 \pm 0.05, \alpha_{3}=1.38 \pm 0.05$, and $\alpha_{4}=1.06 \pm 0.03$. They are 


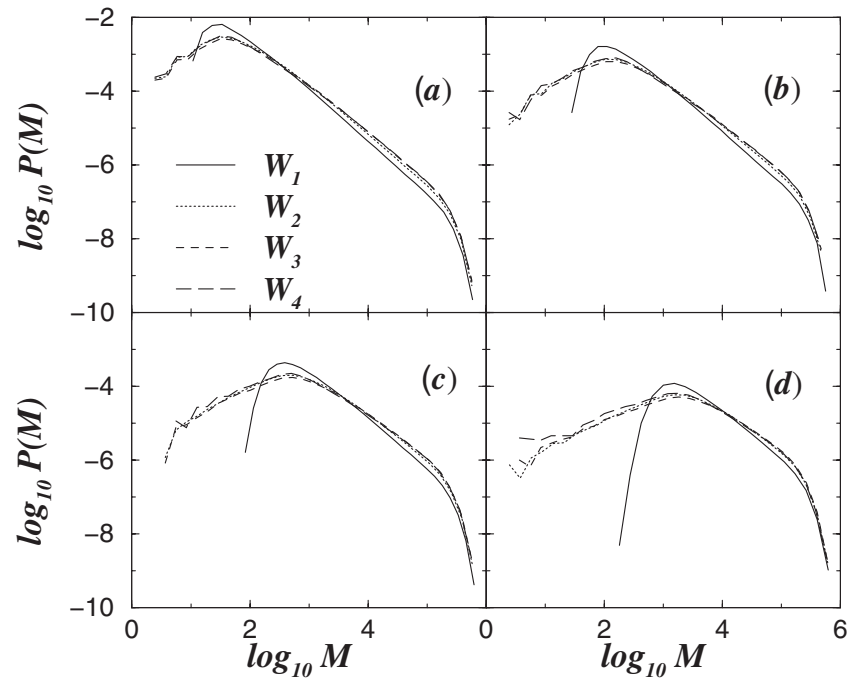

FIG. 4. Log-log plot of $P(M)$ for the case $L=2048$. This result concerns the exploration strategy $B$, in which new wells are bored after the previous one has been exhausted. In this case a well may be located in a saturated region and events with zero recovered mass can be recorded. For the sake of comparison, the events with zero recovered mass were not considered in the statistics. We show the distribution $P(M)$ for (a) $r=8$, (b) $r=16$, (c) $r=32$, and (d) $r$ $=64$. In the case of strategy $B$, the tails of the distributions are weakly affected by the rank of the extraction well. The effect of the parameter $r$ is only to shift the scaling region.

quite close to those of Fig. 2 with a small deviation within the confidence. The value of the parameter $A$ is 0.22 for all four cases. It is worth mentioning that the shape of this cutoff should depend on the size limitations in the oil field. In our model, the field is described as a square lattice but could present more complicated shapes in real oil fields.

Now we investigate the distributions of recovered wells when strategy $B$ is used. In this case, we start with only one extraction well in the beginning of the process. Once this well is reached, a second extraction well is opened and so on. As mentioned before, a well can be opened in a site that has already been invaded and, in this case, the well produces zero recovered mass. In practice, this means that, in strategy $B$, we take a predefined order to bore the sequence of wells while, in strategy $A$, all the wells are initially open, and the order in which they are reached and capped is determined by the invasion process itself. Thus, the mass $M_{1}$ obtained in strategy $B$ can be regarded as the accumulated mass for any of the four stages of strategy $A$. The results for this strategy are illustrated in Fig. 4, where we show the distributions $P_{i}(M)$ for several values of $r$. Typical power laws are obtained again, where the exponent associated with the mass recovered at the first well, $\alpha \simeq 1.39$, corresponds to that of the two-well configuration. The distributions obtained in the following wells present slower decays. The exponent variation with respect to $i$ is significantly reduced, being bounded to the interval $[1.2,1.4]$. Note that, for the sake of a better comparison among the distinct PDF's, we have excluded from the statistics for $i=2,3,4$, all events with null mass. If they are included; the corresponding curves would be displaced downwards, indicating a smaller probability of events in comparison to that of the first well.

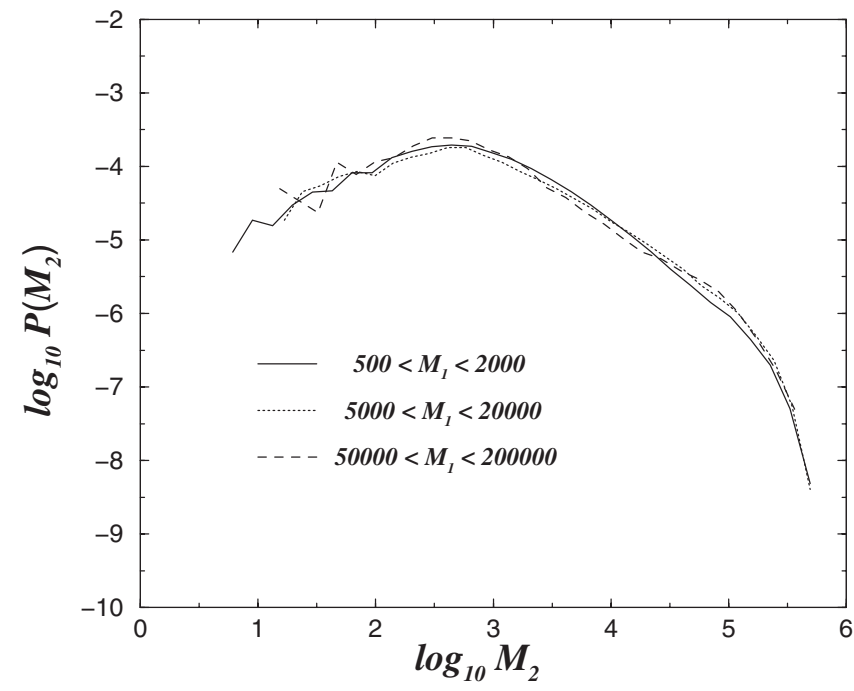

FIG. 5. Log-log plot of the distribution of the mass recovered in the second well when using the exploration strategy $B$. In this figure we study the effect of the amount of oil produced in the first well on the distribution of mass recovered in the second well. These results indicate that, as long as any mass is recovered, the distributions of the recorded mass are not correlated with the previous events. Events with zero mass were not considered in the statistics, but the effect of such events is discussed in Fig. 6. The characteristic lengths for these simulations were $r=32$ and $L=2048$.

As long as four wells are always opened, strategies $A$ and $B$ always result in the same amount of total recovered mass. However, strategy $B$ offers the option of not opening a second or third well depending on the recovered mass obtained up to this point. Alternatively, a decision may be made to bore the following wells in a distant position, with a larger well separation, $r$. These decisions depend on the probability of boring a well on a place already saturated with the invading fluid, what would result in zero production. Interestingly, as long as some mass is recovered, the distribution of recovered oil is independent from the previous events, as shown in Fig. 5. In Fig. 6 we analyze the probability of recovering some mass as a function of the previous recovered mass and the distance between the wells $r$. The inset shows that, when the distance is sufficiently large, the probability of finding the next well over the already invaded region is zero. Given the previous recovered mass $M_{1}$, the typical distance $\xi$ above which it becomes improbable to find an invaded site scales as $M_{1}^{1 / D_{f}}$, where $D_{f} \approx 1.89$ is the fractal dimension of standard invasion percolation [4]. The data collapse resulting from this scaling is shown in the large panel of the figure. Once more, depending on the mass recovered in the previous events, it may be more efficient to choose to open the next well in a more distant position. However, our analysis is restricted to events that do not touch the border. Thus, size limitations of the oil field should also be taken into account to choose the best distance to place the next extraction well.

\section{ANALYTICAL ESTIMATES}

In this section we present some estimates for the PDF's of the amount of extracted oil according to the strategies $A$ and 


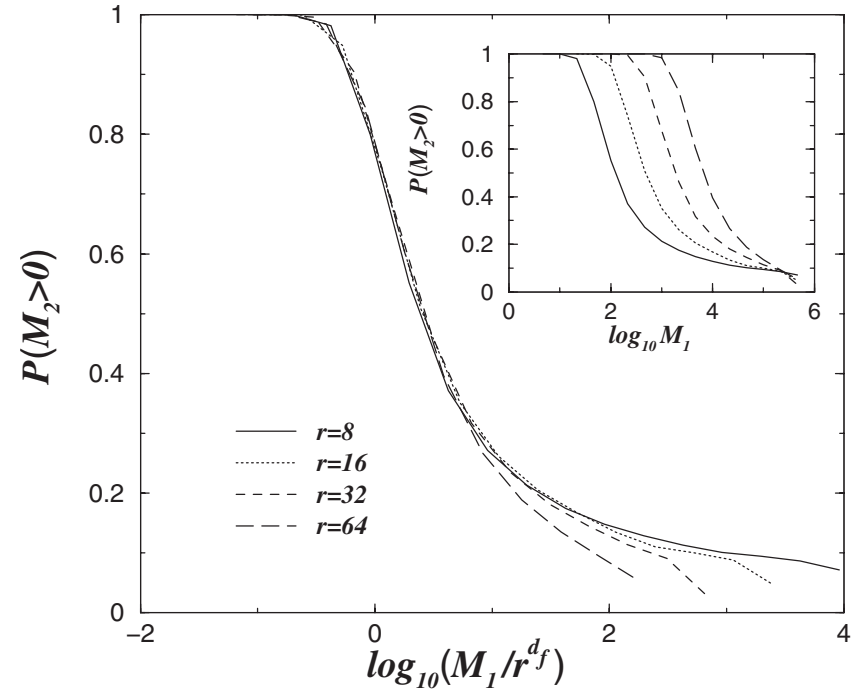

FIG. 6. Probability of having some recovered mass in the second well as a function of the mass obtained in the first well and for different distances $r$. In the inset we show the original data and in the large panel we show the data collapsed by well distance to the power of the fractal dimension $r^{d_{f}}$, with $d_{f}=1.89$.

$B$ discussed in the previous section. Such results, which are obtained under the assumption of independent events, provide a qualitative estimation for the values of the exponents found by means of numerical simulations. For the sake of simplicity, let us first consider the strategy $B$, for the situation of two successively bored extraction wells. We consider the distributions $P_{1}\left(M_{1}\right)$ and $P_{2}\left(M_{2}\right)$ of finding, respectively, the first and second invaded clusters of mass $M_{1}$ and $M_{2}$. To accurately describe this situation, we have to consider single and joint PDF's, respectively, $P_{1}\left(M_{1}\right)$ and $Q_{2}\left(M_{1}, M_{2}\right)$. The probability of finding clusters $M_{1}$ and $M_{2}$ is given by $Q_{2}\left(M_{1}, M_{2}\right) d M_{1} d M_{2}$, while the probability for $M_{2}$ is given by

$$
P_{2}\left(M_{2}\right)=\int_{1}^{\infty} Q_{2}\left(M_{1}, M_{2}\right) d M_{1} .
$$

This procedure can be extended to more general situations where a larger number $N$ of wells are taken into account. Then, new multiple variable PDF's can be introduced in a similar way.

Since new clusters are being formed in the same reservoir (or lattice), a nontrivial dependence of both the joint probability $Q_{N}$ and cluster distribution $P_{N}$ on $P_{i}, 1 \leq i \leq N-1$, should be expected. However, results from the simulations shown in Fig. 5 indicate that all distributions $P_{i}$ are very much alike. Moreover, the maximum amount of sites included in a cluster corresponds roughly to $10 \%$ of all lattice sites. As an approximation, if we consider that the occurrence of clusters $M_{i}$ can be treated as independent events we can write

$$
Q_{2}\left(M_{1}, M_{2}\right) d M_{1} d M_{2}=P_{1}\left(M_{1}\right) P_{1}\left(M_{1}+M_{2}\right) d M_{1} d M_{2},
$$

which, in the general situation of $N$ successively bored wells, becomes

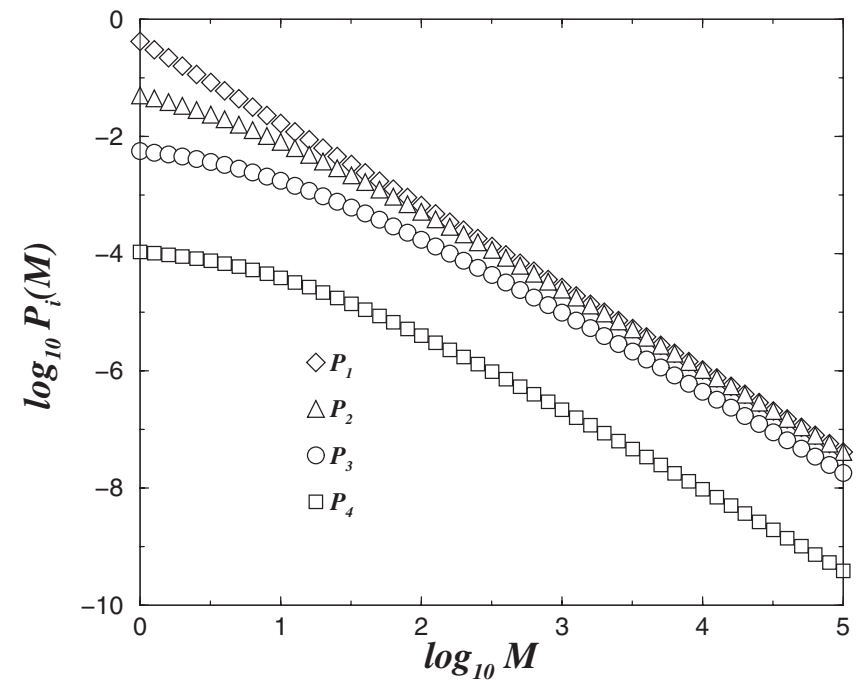

FIG. 7. Distributions $P_{i}, i=1,2,3,4$, obtained according to Eq. (6), when $\alpha=1.4$. They correspond to the distribution of recovered masses obtained with strategy $B$. The curves clearly indicate the effect of polynomial and logarithmic terms on the distributions for low mass values.

$$
Q_{N}\left(M_{1}, \ldots, M_{N}\right) \prod_{i=1}^{N} d M_{i}=\prod_{i=1}^{N} P_{1}\left(\sum_{k=1}^{i} M_{k}\right) d M_{i}
$$

Equation (3) can also be generalized to

$$
P_{N}\left(M_{N}\right)=\int_{1}^{\infty} \prod_{i=1}^{N} P_{1}\left(\sum_{k=1}^{i} M_{k}\right) \prod_{\ell=1}^{N-1} d M_{\ell} .
$$

Results obtained in [8] and reproduced in the previous section indicate that $P_{1}(M) \sim 1 / M^{\alpha}$. Thus, inserting this expression in Eq. (6), we can proceed with the integration over $M_{l}$ to obtain $P_{N}\left(M_{N}\right)$. For $N=2$ and a general exponent $\alpha$, the first integral on $M_{1}$ results in hypergeometrical functions, while for integer values of $\alpha$, these functions reduce to the ratio of polynomials and logarithmic terms. As an example, for $\alpha=2$, we obtain

$$
P_{2}\left(M_{2}\right)=\frac{M_{2}\left(2+M_{2}\right)-2\left(1+M_{2}\right) \ln \left(1+M_{2}\right)}{\left(1+M_{2}\right) M_{2}^{3}} .
$$

For larger values of $N$, we must proceed with numerical methods to obtain the integrals, even for integer values of $\alpha$. It is clear from Eq. (7) that, in the limit of large values of $M_{2}$, $P_{2}\left(M_{2}\right)$ decays with the same exponent $\alpha=2$ as $P_{1}$. However, for finite values of $M_{2}$, lower order terms in Eq. (7) make $P_{2}$ decrease at a slower pace than $P_{1}$. This effect is more evident for smaller values of $\alpha$, as illustrated in Fig. 7, for $\alpha=1.4$. The same remarks are valid also for larger values of $N$, as illustrated in the same figure. As in Fig. 4, the slopes of the curves become only slightly smaller when $i$ increases.

For the strategy $A$, we must replace the distribution $Q_{N}$ by $R_{N, k}\left(M_{1}, \ldots, M_{k}\right), 1 \leq k \leq N$ describing the probability density that, in a field with $N$ bored wells, we obtain clusters of size $M_{k}$ in the time interval limited by two successive events, namely, $k-1$ th and $k$ th wells being capped. Let us here again 
consider the most simple situation, when we have only two extracting wells, say $a$ and $b$. For this configuration we can write

$$
P_{2,1}\left(M_{1}\right)=R_{2,1}\left(M_{1}\right)=P_{a}\left(M_{1}\right) \widetilde{P}_{b}\left(M_{1}\right)+P_{b}\left(M_{1}\right) \widetilde{P}_{a}\left(M_{1}\right),
$$

where $\widetilde{P_{x}}\left(M_{1}\right)$ represents the probability that well $x$ will not be reached by the percolating cluster for any value of 1 $\leq M \leq M_{1}$,

$$
\tilde{P}\left(M_{1}\right)=1-\int_{1}^{M_{1}} P_{1}(M) d M=M_{1}^{-\alpha+1} .
$$

As the two extraction wells are equivalent, the two terms in Eq. (8) contribute equally, which leads us to

$$
R_{2,1}\left(M_{1}\right)=2(\alpha-1) / M_{1}^{2 \alpha-1} .
$$

If we proceed along the same lines, it is possible to conclude that, for any number $N$ of wells,

$$
P_{N, 1}\left(M_{1}\right)=R_{N, 1}\left(M_{1}\right)=N(\alpha-1) / M_{1}^{N \alpha-N+1},
$$

from which we obtain the PDF's $R_{N, k}$ by performing the following integrals:

$$
\begin{aligned}
P_{N, k}\left(M_{k}\right) & =\int_{1}^{\infty} R_{N, k}\left(M_{1}, \ldots, M_{k}\right) \prod_{i=1}^{k-1} d M_{i} \\
& =\int_{1}^{\infty} \prod_{i=1}^{k}\left(\frac{1}{\sum_{j=1}^{1} M_{j}}\right)^{(N-i+1) \alpha-N+i} \prod_{\ell=1}^{k-1} d M_{\ell} .
\end{aligned}
$$

Again, it is possible to integrate Eq. (12) only for some special values of $\alpha$ and $k$. For example, when $\alpha=2$ and $k=1$, the integral leads to

$$
P_{2,2}\left(M_{2}\right)=\frac{M_{2}\left(M_{2}^{2}-3 M_{2}-6\right)+6\left(1+M_{2}\right) \ln \left(1+M_{2}\right)}{2\left(1+M_{2}\right) M_{2}^{4}},
$$

indicating that, in the large mass limit, $P_{2,2} \sim 1 / M_{2}^{2}$. Results for $N=4,1 \leq k \leq 4$, and $\alpha=1.4$, obtained by numerically integrating Eq. (12), are illustrated in Fig. 8. The curves clearly indicate the effect of polynomial and logarithmic terms on the distribution for low mass values. As a consequence, before reaching the asymptotic region, the slope of the curves is less negative than in the bare tails. These are described by power laws and, for arbitrary $N$ and $k$ can be expressed by

$$
P_{k}\left(M_{k} ; N\right) \sim 1 / M_{k}^{(N-k)(\alpha-1)-\alpha} .
$$

The behavior displayed in Fig. 8 is similar to that obtained by our numerical simulations, i.e., the exponents become less negative when $k \rightarrow N$. For $N=4$, the values of $\alpha_{i}, i$ $=1,2,3,4$ are 2.6, 2.2, 1.8, and 1.4, respectively. The relative deviations of these values to those obtained by the numerical simulations become larger as $i$ increases. They may be explained by the fact that in our analytical treatment we did not consider correlations among the successive events.

\section{DISCUSSION}

In this work we investigate the statistics of the size of invasion percolation clusters generated between a single

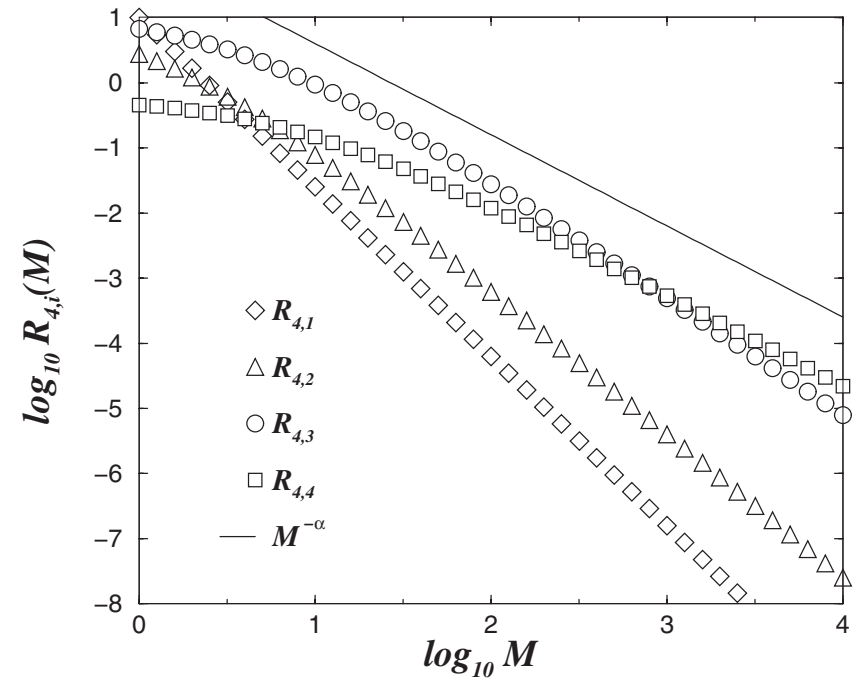

FIG. 8. Distributions $R_{4, i}, i=1,2,3,4$, obtained according to Eq. (12), when $\alpha=1.4$. The exponents describing the amount of recovered masses depend strongly on $i$, in a similar way as obtained in the simulations for strategy $A$, shown in Fig. 2. Note also that, in the finite-size region, the slope is less pronounced than in the asymptotic region. The distribution $P_{1}$ is also included, for the purpose of eye guiding.

starting point and four possible end points. The motivation of our work comes from the problem of oil recovering in exploration fields. We show that, when fixing the position of the extraction wells, the distribution of recovered oil in each of the extraction wells depends on whether they are opened simultaneously or in a predefined sequence, while the total amount of recovered oil does not depend on the particular exploration strategy. Additionally, we find that, in the sequential strategy $(B)$ the recovery distribution has the same exponent controlling the decay of the tail, independent of which of the subsequent wells is taken, provided they are not placed on an already invaded site. Thus, the decision to bore further wells after previous wells have been reached should depend on the total recovered mass up to that point. Our results indicate that the production is more efficient when posterior wells are placed at a distance larger than the characteristic size of the invaded cluster, which is proportional to $M^{1 / D_{f}}$. However, practical limitations should be taken into account to assure that the well is placed within the region covered by the oil field. In our analysis we do not take into account the limiting boundary of the field. It is also important to mention that, with this power-law decay, the mean recovered mass diverges in the limit of infinite-size systems. This divergence would not appear in real systems where limiting factors are always present. Finally, analytical estimates for the mass distribution, based on the assumption of independent events, reproduce the qualitative behavior of the numerical simulations. These results could be relevant for the decision strategies in oil exploration fields.

\section{ACKNOWLEDGMENTS}

This work was supported by CNPq (CT-PETRO/CNPq), CAPES, FINEP, FAPESB, and FUNCAP/CNPq/PPP. 
[1] J. Bear, Dynamics of Fluids in Porous Materials (Elsevier, New York, 1972).

[2] F. A. Dullien, Porous Media-Fluid Transport and Pore Structure (Academic, New York, 1979).

[3] J. Feder, Fractals (Plenum Press, New York, 1988).

[4] D. Stauffer and A. Aharony, Introduction to Percolation Theory (Taylor Francis, Philadelphia, 1994).

[5] Fractals and Disordered Systems, 2nd ed., edited by A. Bunde and S. Havlin (Springer-Verlag, New York, 1996).

[6] Y. Lee, J. S. Andrade, Jr., S. V. Buldyrev, N. V. Dokholyan, S. Havlin, P. R. King, G. Paul, and H. E. Stanley, Phys. Rev. E 60, 3425 (1999).

[7] D. Wilkinson and J. F. Willemsen, J. Phys. A 16, 3365 (1983).

[8] A. D. Araújo, T. F. Vasconcelos, A. A. Moreira, L. S. Lucena, and J. S. Andrade, Jr., Phys. Rev. E 72, 041404 (2005).

[9] P. R. King, S. V. Buldyrev, N. V. Dokholyan, S. Havlin, E. Lopez, G. Paul, and H. E. Stanley, Physica A 314, 103 (2002).

[10] R. Chandler, J. Koplik, K. Lerman, and J. F. Willemsen, J. Fluid Mech. 119, 249 (1982).

[11] M. Sahimi, Flow and Transport in Porous Media and Fractured Rock (VCH, Boston, 1995), and the extensive references therein.

[12] M. Murat and A. Aharony, Phys. Rev. Lett. 57, 1875 (1986).

[13] B. Clark and R. Kleinberg, Phys. Today 55, 48 (2002).

[14] A. D. Araújo, A. A. Moreira, R. N. Costa Filho, and J. S. Andrade, Jr., Phys. Rev. E 67, 027102 (2003).

[15] A. D. Araújo, J. S. Andrade, Jr., and H. J. Herrmann, Phys. Rev. E 70, 066150 (2004). 